\title{
Jobs and skills in Industry 4.0: an exploratory research
}

\author{
Marta Pinzone ${ }^{1}$, Paola Fantini ${ }^{1}$, Stefano Perini ${ }^{1}$, Stefano Garavaglia $^{1}$, Marco Taisch $^{1}$, \\ Giovanni Miragliotta ${ }^{1}$ \\ ${ }^{1}$ Politecnico di Milano, Department of Management Engineering, via Lambruschini 4/b, 20156 \\ Milan, Italy \\ \{marta.pinzone, paola.fantini, \\ stefano.perini, stefano.garavaglia, marco.taisch, \\ giovanni.miragliotta\}@polimi.it
}

\begin{abstract}
Industry 4.0 is at the center of the current debate among manufacturing leaders, industrial practitioners, policy makers and researchers. Despite the increasing attention paid to changes in jobs and skills generated by Industry 4.0, research in this domain is still scarce. Our study focuses on the evolution of technical skills in the context of Industry 4.0 and it provides qualitative insights gained from an on-going collaborative research project involving a variety of manufacturing stakeholders in Northern Italy (e.g., manufacturing companies, industrial associations, academic and education experts, recruiting companies, IT providers, consultants, etc.). Our findings contributes to shed light on manufacturing skill needs linked to Industry 4.0, setting the stage for future research on the topic and providing companies, policy makers and education stakeholders with first indications to detect skill gaps and initiate competence development.
\end{abstract}

Keywords: Industry 4.0, Jobs, Skills, Competence.

\section{Introduction}

The evolution of jobs and skills for the successful implementation of Industry 4.0 is of significant contemporary interest and importance to both researchers [1], policy makers [2] and practicing managers [3].

The introduction of the latest automation and digital technologies in manufacturing - such as Cyber-Physical Systems, the Internet of Things, cloud computing and Big Data - is envisaged to significantly affect work processes and the work environment [4]. Employee jobs are expected to change in terms of content and new types of job are also being created. As a consequence, novel skill requirements are foreseen [2] [5].

Notwithstanding the increasing attention paid to the topic, research on the changes in the jobs and skills required by Industry 4.0 is still emerging. So far, research (e.g., [1] [6]) were mostly theoretical and provided a generic overview on Industry 4.0-related competencies, without articulating them according the specificities of the different organizational areas where technologies are being introduced.

Consequently, significant gaps remain on how job profiles will evolve and what types of skills will be relevant and in demand in Industry 4.0 [7]. 
In this paper, we aim at contributing to fill the above-mentioned gaps by providing a fine-grained set of technical skills related to 5 organizational areas affected by Industry 4.0 - i.e. operations management, supply chain management, product-service innovation management, data science management and IT-OT integration management.

We focus on technical skills as they are crucial to the understanding of and participation in the new Industry 4.0 workplaces.

In doing so, we make two main contributions to our knowledge. First, our study advance current research shedding further light on technical skills for Industry 4.0. Second, our study goes beyond previous research, since it combines findings from the literature with the expert knowledge of different manufacturing stakeholders.

Our results also contributes to practice by providing business, government and education with new relevant insights for the identification of re-skilling, up-skilling needs and the development of the future industrial human capital.

The rest of the paper is structured as follows. In the next section, we provide an overview of extant models and research on skills for Industry 4.0. Subsequently, we describe the methodology adopted. We then present the set of technical skills coming from our exploratory research. Finally, we outline our contributions, the limitations of our study and propose some suggestions for future research.

\section{$2 \quad$ Related work}

Competency models refer to collections of knowledge, skills, abilities, and other characteristics that are needed for effective performance in the jobs, job families or functional areas [8] [9]. During last years, several works were carried out in order to define competency models for manufacturing, ICT and Industry 4.0. The most significant ones for the scope of our study are summarised in the followings.

The U.S. Employment and Training Administration (ETA) developed a competency model framework depicted in a pyramid graphic with nine tiers. Each tier is comprised of blocks representing the skills, knowledge, and abilities essential for successful performance. The general framework was then tailored - among others - to the U.S. Advanced Manufacturing, Automation, Aerospace and Mechatronics industries [10]. Linked to the ETA framework is the $\mathrm{O} *$ NET program, which provides a description of the mix of knowledge, skills, and abilities, as well as the tasks, tools and technologies required by different occupations in the U.S. [11].

With regard to ICT-related competences, the European "e-Competence Framework" provides 40 competences, including skills and knowledge requirements, which can be used across Europe [12]. Similarly, the EDISON project is working on the development of a "Data Science Competences Framework" that includes: competence groups, big data and analytics tools and programming languages [13].

It is worth noting that the above-mentioned frameworks were not developed with a specific focus on Industry 4.0 but they represent a useful starting point for the identification of relevant skills and the analysis of their evolution in Industry 4.0.

Regarding Industry 4.0, [1] proposed a skillset for the production worker of the future. The identified competences were clustered into two categories: technical and personal 
qualifications and skills. Among the technical ones, IT knowledge and abilities, organizational and processual understanding, ability to interact with modern interfaces (human-machine / human-robot) were identified as a "must have" in the future Industry 4.0 scenario. Additionally, [6] presented the development of a generic competence model for Industry 4.0, including technical, methodological, social and personal competencies. The technical skills reported in the study are: state-of-the-art knowledge, technical skills, process understanding, media skills, coding skills, understanding IT security.

Despite the important insights generated by the two papers, they addressed skills in a generic way, without articulating and differentiating them for different organizational areas or job families.

\section{$3 \quad$ Method}

The study used a three-steps process based on: literature review, focus groups and in-depth interviews. First, the literature review aimed at identifying existing organizational areas of advanced manufacturing and the technical skills related to each of them. Then, technical skills classifications for the manufacturing field were analyzed. Different sources were used, namely scientific papers as well as reports and classifications provided by national and international bodies (e.g. the O*NET-SOC 2010 Taxonomy [9] sponsored by the US Department of Labor/Employment and Training Administration and the European e-Competence Framework [10] sponsored by the European Commission).

The organizational areas and technical skills identified through the literature review were, then, used as an input for two subsequent focus groups [12]. The two focus groups aimed at refining and further specifying the initial list of skills by collecting new insights and ideas from recognized manufacturing experts. In particular, the first focus group involved 20 participants, with representatives from Industry 4.0-related technology providers, academia, research centers and national institutions, while the second one, involving 50 participants, specifically addressed manufacturing companies moving to Industry 4.0. All the stakeholders involved were from Northern Italy. During the focus groups, all participants' ideas were first collected on post-its and posters, and then categorized by means of an Affinity Diagramming approach [14]. The focus group discussions were managed by the authors of the paper by following the principles identified by Axelrod [15].

Finally, based on the results of the two focus groups, in-depth interviews with further relevant stakeholders from both technology providers and manufacturing companies were carried out to check and refine the technical skills in each of the five organizational areas. The results obtained after the interviews are reported in the following paragraph.

\section{$4 \quad$ Results}

The following sub-sections summarize the final sets of technical skills for Industry 4.0 in each of the 5 organizational areas under investigation: 1) Operations Management, 
2) Supply Chain Management, 3) Product-Service Innovation Management, 4) Data Science Management, 5) IT-OT Integration Management.

\subsection{Operations Management}

- Defining a roadmap to the adoption of Industry 4.0 technologies aiming to continuous improvement;

- Analysis, modelling and simulation of production based on big data from sensors and devices;

- Use of digital devices (e.g. tablets, smartphones, smartwatches) for production monitoring and control;

- Programming and use of collaborative robots;

- Use of additive manufacturing technologies;

- Management of human resources, interconnected through digital devices;

- Design of predictive maintenance systems (sensors, data flows and analytics);

- Remote system monitoring and supervision of maintenance interventions;

- Use of virtual and augmented reality for instruction and support of maintenance interventions on-field;

- Defining systems for monitoring maintenance services to support the definition (e.g. service level agreements) and management of maintenance service contracts, by using big data from the sensors.

\subsection{Supply Chain Management}

- Design and building Digital Supply Networks;

- Management of Concurrent Digital Supply Networks;

- Use virtual design for business process;

- Analysis of big data (e.g. sentiment analysis) to predict market behaviour and other phenomena impacting the supply chain;

- Development of IT strategies to support Supply chain Management (e.g. collaboration platforms on the cloud and Internet of Things);

- Real time management leveraging monitoring and tracking technologies;

\subsection{Product-Service Innovation Management}

- Research, analysis and use of innovative materials (e.g., shape-memory materials, composites) and/or production processes (e.g., additive manufacturing);

- Design of smart products (integration of sensors, antennas, chips and other components);

- Design of the service model (functionalities, interactions) based on the product-service platform;

- Joint design of product and service, integration with the enterprise IT systems. 


\subsection{Data Science Management}

- Design and implementation of Big Data architectures and software platforms (e.g. e Hadoop o Data Lake);

- Design of data and workflow models;

- $\quad$ Big Data management, use of cloud computing and data storage;

- Development of applications and tools for Big Data analytics (e.g. R, Python);

- Big Data analytics (e.g. machine learning, Bayesian classifiers, deep learning);

- Info-graphics for intuitive and engaging interpretation of data analytics (e.g. maps, charts, diagrams);

- User experience design.

\subsection{IT-OT Integration Management}

- Development of a strategic roadmap for the integration of information technologies (IT) and operations technologies of industrial automation (OT), in alignment with business needs;

- Implementation of IT architectures, platforms and components oriented towards Industry 4.0;

- Selection, specification and integration of embedded devices, cyber-physical systems and advanced Human-Computer-Interfaces (e.g. interface for mobile applications, Virtual and Augmented Reality)

- Implementation of IT networks enabling real-time connection of robot, machines, products and people;

- Selection and application of data communication protocols (IIoT, cloud, cybersecurity, Big Data) and Industry 4.0-related standards;

- Use of modelling tools to generate digital twins of manufacturing systems and simulate "what if scenarios";

- Use of graphic modelling tools to specify, analyse, design and verification of complex systems, including hardware and software components;

- Design of structured strategies for and management of cybersecurity, data privacy and safety.

\section{$5 \quad$ Discussion and conclusions}

Even though manufacturing is rapidly moving towards Industry 4.0, there is still a lack of knowledge about the consequent evolution of job profiles and skills, and how manufacturing companies should deal with the skills gap that is being created.

For this reason, the current paper presented the results of one of the first exploratory studies aimed at outlining the new set of Industry 4.0 skills required for the successful implementation of the approach. In particular, the work aimed at reaching a twofold objective. On one hand, the identification of the latest needs of manufacturing in terms of Industry 4.0 skills. On the other hand, an attempt to anticipate the needs of companies in the near future. Therefore, the results obtained can constitute a sound basis for a wide 
range of different actions, e.g. recruitment and training of workers, organizational design, business process improvement.

Three main limitations can be identified for the current study. First, the research was based on a qualitative methodology, which allowed to develop a structured list of technical skills for Industry 4.0. To strengthen the proposed model, a survey should be used in order to further validate the list by means of a quantitative approach. In addition, the survey will be able to provide further information about the maturity level of companies in terms of skill levels for Industry 4.0, and about the potential differences between small, medium and large enterprises. Second, given its exploratory nature, the study was based on the contributions of Northern Italy representatives. The findings should be expanded through the access to a wider European audience, in order to identify the possible cultural, systemic and industrial divergences in the perception and solution of the Industry 4.0 skills issue. Finally, the present study is focused on skills, while future research could expand the scope of the analysis by investigating knowledge, abilities and attitudes in order to create a thorough Industry 4.0 competency model,

In conclusion, despite the limitations this study has, it advances both research and practice by providing a fine-grained set of Industry 4.0-related technical skills for 5 organizational areas.

\section{Acknowledgement}

This work has been partly funded by the European Union's Horizon 2020 research and innovation programme (grant agreement $\mathrm{n}^{\circ} 680633$ ).

The Authors also wish to thank the "Industry4.0" Observatory of the School of Management of Politecnico di Milano and all the companies participating in the focus groups for their valuable inputs to the work.

\section{References}

[1] VDI/ASME, «A Discussion of Qualifications and Skills in the Factory of the Future: A German and American Perspective,» 2015.

[2] J. Smit, S. Kreutzer, C. Moeller e M. Carlberg, «Industry 4.0,» 2016.

[3] The Boston Consulting Group, «Man and Machine in Industry 4.0,» 2015.

[4] G. Chryssolouris, M. Dimitris e M. Dimitris, «Manufacturing Systems: Skills \& Competencies for the Future,» in Procedia CIRP, 2013.

[5] E. A. Hartmann e M. Bovenschulte, «Skills Needs Analysis for "Industry 4.0" Based on Roadmaps for Smart Systems,» in Using Technology Foresights for Identifying Future Skills Needs. Global Workshop Proceedings, Moskow, 
SKOLKOVO Moscow School of Management \& International Labour Organization, 2013, pp. 24-36.

[6] F. Hecklaua, M. Galeitzkea, S. Flachsa e H. Kohlb, «Holistic approach for human resource management in Industry 4.0,» in Procedia CIRP, 2016.

[7] B. Dworschak e H. Zaiser, «Competences for cyber-physical systems in manufacturing - first findings and scenarios,» in Procedia CIRP, 2014.

[8] D. Mavrikios, N. Papakostas, D. Mourtzis e G. Chryssolouris, «On industrial learning and training for the factories of the future: a conceptual, cognitive and technology framework,» Journal of Intelligent Manufacturing, vol. 24, n. 3, pp. 473-485, 2013.

[9] . M. A. Campion, A. A. Fink, B. J. Ruggeberg, L. Carr, G. M. Phillips e R. B. Odman, «Doing competencies well: Best practices in competency modeling,» Personnel Psychology, vol. 64, n. 1, pp. 225-262, 2011.

[10] ETA Industry Competency Initiative, «Competency Model Clearinghouse,» [Online]. Available: https://www.careeronestop.org/CompetencyModel/.

[11] O*NET Resource Center, «O*NET Resource Center,» [Online]. Available: https://www.onetcenter.org/.

[12] CEN/TC 428, «e-Competence Framework (e-CF) - A common European Framework for ICT Professionals in all industry sectors - Part 2: User Guide,» 2016.

[13] Y. Demchenko, A. Belloum, W. Los, T. Wiktorski, . A. Manieri, H. Brocks, J. Becke, D. Heutelbeck e M. Hemmje, «EDISON Data Science Framework: A Foundation for Building Data Science Profession For Research and Industry.,» in 2016 IEEE 8th International Conference on Cloud Computing Technology and Science, 2016.

[14] A. Goldman, «The group depth interview,» Journal of Marketing, vol. 26, n. 3, pp. 61-68, 1962.

[15] G. May, B. Stahl e M. Taisch, «Energy management in manufacturing: Toward eco-factories of the future - A focus group study,» Applied Energy, vol. 164, pp. 628-638, 2016.

[16] J. Kawakita, «The KJ method - A scientific approach to problem solving,» Kawakita Research Institute, Tokyo, 1975.

[17] M. Axelrod, «10 essentials for good qualitative research,» Market News, vol. 8, n. 17, pp. 10-11, 1975. 